\title{
Tiers et objectivité sociale chez Georg Simmel
}

\begin{abstract}
Avertissement
Le contenu de ce site relève de la législation belge sur la propriété intellectuelle et est la propriété exclusive de l'éditeur.

Les œuvres figurant sur ce site peuvent être consultées et reproduites sur un support papier ou numérique sous réserve qu'elles soient strictement réservées à un usage soit personnel, soit scientifique ou pédagogique excluant toute exploitation commerciale. La reproduction devra obligatoirement mentionner l'éditeur, le nom de la revue, l'auteur et la référence du document.

Toute autre reproduction est interdite sauf accord préalable de l'éditeur, en dehors des cas prévus par la législation en vigueur en Belgique.
\end{abstract}

\section{Référence électronique}

Grégoire Lits (2009), « Tiers et objectivité sociale chez Georg Simmel », Emulations.

URL : http://shortlinks.revue-emulations.net/xnpvt

Éditeur : Émulations - Revue des jeunes chercheurs en sciences sociales http://www.revue-emulations.net 


\section{Tiers et objectivité sociale chez Georg Simmel}

Grégoire Lits

Résumé : La notion de tiers apparaît dans différents textes de Georg Simmel. Sans être systématiquement conceptualisée par l'auteur, elle permet de comprendre la vision du monde social qui sous-tend la sociologie qu'il propose. A partir de l'analyse de deux chapitres de Sociologies, nous pourrons montrer que la société pour Simmel ne se réduit pas à une somme d'interactions mais possède des aspects extérieurs - holistes - rassemblant les individus. Dans une seconde partie la notion de tiers est examinée quant à son intérêt pour l'étude des sociétés contemporaines mondialisées en établissant, à partir des travaux de Vincent Descombes sur les significations partagées, une différenciation entre deux types de tiers sociaux.

Mots-clés : Simmel, tiers, relations sociales, relations intersubjectives, holisme.

Abstract: The concept of "third" - that is, a social presence outside person-to-person relationships - appears in various texts of Georg Simmel. Without being systematically conceptualized by the author, this notion nonetheless helps us to understand which idea of the social world lies beneath his sociology. From the analysis of two chapters of Simmel's Sociology, we clarify this notion in a way to show that instead of reducing society to a sum of interactions, Simmel's sociology rather implies holistic aspects. In a second part, we argue that such a notion of the "third" is useful in many ways for the study of contemporary global societies, in the line of Vincent Descombes's holistic conception of society

Keywords: Simmel, Third, Social Relations, Intersubjective Relations, Holism.

\section{Introduction ${ }^{1}$}

On ne connaît souvent de la sociologie de Georg Simmel que quelques grands thèmes de ces travaux liés par exemple à la ville ou à la place de l'argent dans la société moderne. Sa sociologie formelle méconnue sous bon nombre d'aspects dans la littérature francophone, ou du moins dans les recueils d'histoire des idées sociologique, est très souvent réduite à un interactionnisme primordial sources d'inspiration des Goffman, Garfinkel ou Boudon.

Certains textes importants et pourtant moins connus du sociologue présentent cependant une vision du monde sociale particulière qui est loin de se réduire à un interactionnisme considérant que «La Société » n'existe pas et qu'il n'y a que des individus pris dans des actions réciproques. A l'inverse il est possible de montrer que la vision que Simmel construit contient les éléments d'une théorie holiste des

\footnotetext{
1. Cet article a été réalisé dans le cadre d'un DEA Interuniversitaire en sociologie poursuivi à l'université catholique de Louvain et à l'Ecole des Hautes Etudes en Sciences Sociales à Paris. Il résulte de travaux de recherche réalisés sous la direction des professeurs Bernard Francq (UCL/CADIS) et Louis Quere (EHESS/CEMS) dans le cadre du travail de fin d'étude et du séminaire « Normes, conventions et institutions ». Je remercie ces deux professeurs pour leurs conseils.
}

Émulations - Revue des jeunes chercheurs en sciences sociales 
relations sociales. En d'autre terme il est possible de montrer que pour Simmel il existe bel et bien pour qu'il y ait société des éléments communs liant les individus qui leurs sont extérieurs et que ces éléments doivent être pris en compte par le sociologue qui étudie les formes de socialisation.

Cet article propose, à partir de la lecture de deux textes publiés en 1896 et en 1902 dans l'American journal of Sociology (respectivement «Superiority and subordination as subject-matter of sociology » (1896) et "The number of members as determining the sociological form of the group" (1902)), de reconstruire une vision du monde social développée par Simmel à partir de la notion de tiers qu'il développe ${ }^{2}$ dans ces deux textes.

Une fois la notion de tiers social analysée, il sera possible de montrer à partir de la synthèse des débats sur la perspective holiste réalisée par Vincent Descombes que la vision du monde sociale développée par Simmel correspond aux critères d'une analyse holiste de la société.

\section{Différentes acceptations des tiers sociaux}

L'œuvre de Simmel, comme on le sait, se caractérise sans doute par un certain manque de régularité du point de vue de la construction conceptuelle. Il ne propose en effet que très rarement des considérations théoriques abouties sur telle ou telle notion, pour se concentrer principalement sur l'analyse des formes des actions réciproques. Le concept de tiers qu'il propose n'échappe pas à la règle. Il faudra donc reconstituer à partir de diverses parties de son œuvre la conception du monde social que Simmel pose lorsqu'il parle de tiers.

Ce flou conceptuel se ressent très vite. Dans l'article The number of members as determining the sociological form of the group, nous trouvons ainsi un paragraphe très intéressant où Simmel propose d'étudier la signification du chiffre trois dans les relations (Simmel, 1999 : 128-152).

Il propose d'analyser les formes particulières que prennent les actions réciproques impliquant trois partis principaux. Premier constat : ce que Simmel désigne quand il parle de groupe composé de trois parties n'est pas réellement clair. Dans un premier sens, il semble que ce qui intéresse Simmel est de décrire différents types de relations qui peuvent concrètement lier trois individus dans une socialisation. Le tiers serait donc, dans un premier sens, le troisième individu qui, interférant par sa qualité dans une interaction de deux personnes, viendrait donner une nouvelle forme, prévisible - puisque ne pouvant prendre qu'une des trois formes décrites par Simmel - à l'interaction en cours ${ }^{3}$. Cette première conception du tiers correspond en fait, comme l'a montré André Berten, à la conception commune du tiers, telle que présentée dans les dictionnaires Robert ou Larousse. Comme il le note :

De la façon la plus générale, l'usage commun [de la notion de tiers] signifie qu'une " troisième personne " s'ajoute à la relation duelle ou à une relation intragroupe, et interfère dans cette relation duelle ou groupale. (Berten, $2005: 40-41$ )

Dans un premier sens, donc, la notion de tiers au sens simmelien pourrait être celle-ci. Il s'agirait d'un troisième individu ou, par extension, d'un troisième groupe qui influencerait la relation de deux autres individus ou de deux autres groupes.

Une telle interprétation de la conception simmelienne a été poussée le plus loin par Théodore Caplow. Selon lui : «l'interaction est le processus social de base grâce auquel les individus et les groupes exercent une influence les uns sur les autres, et c'est un processus triangulaire - ou triadique - parce

2. Ces articles ont par la suite été repris par Simmel dans son ouvrage Sociologie publié en Allemand pour la première fois en 1908 et dont une traduction intégrale a été publié par les PUF en 1999. L'ensemble des citations renvoie à l'édition 1999 des PUF.

3. Simmel nous dit en effet que «la triade en tant que telle engendre trois formes typiques de regroupement» (Simmel, 1999 : 131). Ces trois formes sont celles 1/ du juge impartial et du médiateur ; 2/ le tertius gandens : le tiers qui fait «de l'action réciproque entre les parties et lui-même un moyen au service de ses fins propres » (p. 139) ; et 3/ le divide et impera: «le tiers qui suscite la querelle intentionnellement pour acquérir une position dominante » (p. 146). Notons que nous nous intéresserons uniquement à la première de ces trois formes qui est celle où Simmel présente le plus d'éléments permettant de proposer une seconde conception du tiers.

Émulations - Revue des jeunes chercheurs en sciences sociales 
qu'il subit toujours l'influence d'un public, présent ou proche» (Caplow, 1971 : p.10). Caplow mit alors sur pied un programme de recherche visant, dans une perspective interactionniste directement inspirée de Simmel, à démontrer que la triade est le matériau de base à partir duquel se construisent toutes les organisations. Son ouvrage Deux contre un visera à l'élaboration de formules permettant, en fonction de la force ou de la puissance des trois individus ou groupes en présence, la prédiction de la forme que prendra l'interaction triadique.

Cette interprétation est correcte. Cependant s'y limiter ne permet pas de percevoir la richesse de la vision simmelienne du monde social. Tel en atteste l'extrait suivant, qui permettra d'introduire une seconde interprétation de la notion de tiers dans un sens simmelien :

La relation commune d'éléments isolés envers une puissance extérieure à eux, constituant le fondement de leur unification, est un fait sociologique extrêmement important - depuis l'alliance entre États dans le but de se défendre contre un ennemi commun, jusqu'à l'Église invisible qui rassemble tous les croyants en une unité fondée sur la relation égale de chacun à un seul Dieu. Mais cette médiation d'un troisième élément constituant une société devra être traitée dans un autre contexte. Car le troisième élément est ici à une telle distance des deux autres qu'il n'y a pas de véritables actions réciproques sociologiques qui réuniraient les trois éléments, mais au contraire des configurations duales : ou bien c'est la relation de ceux qui se réunissent qui est sociologiquement mise en question, ou bien la relation de l'unité qu'ils constituent avec le centre d'intérêts qu'ils ont en face d'eux. Mais il s'agit d'éléments si proches ou si près de l'être qu'ils constituent un groupe, de façon permanente ou momentanée. (Simmel, 1999 : 131-132.)

Cette citation montre que Simmel lui-même distingue deux types de tiers qui viennent à jouer un rôle dans la vie sociale. Le sociologue peut, d'une part, s'intéresser aux tiers entendus dans leur acception commune et analyser « la relation de ceux qui se réunissent», en formant donc une triade. Mais il peut également étudier «la relation de l'unité qu'ils constituent [ceux qui se réunissent] avec le centre d'intérêts qu'ils ont en face d'eux». Dans cette seconde acception, le tiers est donc, à l'instar de l'Église, un troisième élément, invisible, qui a pour rôle ou pour fonction de rassembler des individus différents en une unité. Dans cette acception, le tiers est l'élément médiateur qui permet la constitution de la société et qui se trouve au-delà de l'interaction en tant que telle entre plusieurs individus. Pour reprendre les mots de Simmel, : «le troisième élément est ici à une telle distance des deux autres qu'il n'y a pas de véritables actions sociologiques qui réuniraient les trois éléments, mais au contraire des configurations duales ». Nous sommes, avec cette phrase, dans une conception du tiers toute différente de celle proposée par Caplow. Elle nous place dans une perspective où la base de l'analyse ne serait plus l'interaction concrète liant trois individus dans une situation particulière, mais bien la relation liant plusieurs individus à une entité invisible, non matérielle, présente dans les actions réciproques et qui permet aux individus de se lier et de former une « unité ».

\subsection{Le tiers : médiateur et objectivité}

C'est cette seconde acception qui va être développée dans cet article. Il faut pour cela définir clairement ce que Simmel veut dire quand il parle de tiers dans ce sens. Nous pouvons trouver des éléments de réponse dans la suite de l'article sur l'influence du nombre sur la forme des interactions, ainsi que dans l'article «Superiority and subordination as subject-matter of sociology ».

Nous pouvons ainsi dégager plusieurs caractéristiques, qui selon Simmel, désignent le tiers. Nous verrons que le rôle du tiers est d'être médiateur et que le mécanisme dont il procède consiste en l'objectivation d'énergie subjective. Voyons d'abord en quoi le tiers est médiateur.

La fonction du tiers, qui consiste à servir de médiation entre deux extrêmes, peut se répartir entre plusieurs ; ici ce n'est en quelque sorte qu'un élargissement, ou encore un affinement de la mise en œuvre technique du principe. Celui-ci, le changement qui décide intérieurement de la configuration, se réalise toujours dès que vient s'ajouter le troisième parti. (Ibid., p.131)

Émulations - Revue des jeunes chercheurs en sciences sociales 
La fonction du tiers est qu'il sert de médiation entre deux éléments différents. Qu'est-ce que Simmel entend par-là? La réponse à cette question comporte deux éléments. Il semble tout d'abord, comme on le voit dans cet extrait, que le principe de la médiation consiste en le fait qu'un changement dans la configuration d'une interaction soit toujours lié à la présence d'un tiers. Le tiers est médiateur en ce qu'il doit être présent pour que se configure une interaction particulière (que ce tiers soit un individu ou un élément extérieur à la simple action réciproque). Dans un second temps, si l'on pousse ce principe jusqu'au bout, il semble que pour Simmel le rôle du tiers soit finalement d'être l'élément qui lie les deux individus prenant part à l'interaction en une unité. Tel en atteste l'extrait suivant :

Mais le vrai résultat, c'est que c'est seulement l'arrivée du troisième élément qui ferme en fait le cercle, en liant les deux autres l'un à l'autre. Cela peut prendre deux formes : ou bien l'existence du troisième élément fonde directement ou consolide la liaison des deux autres - comme par exemple quand la naissance d'un enfant accroît l'amour entre les époux ou du moins celui de l'homme pour la femme - ou bien la relation de chacun des deux au troisième instaure entre eux une relation nouvelle et indirecte - de même que les soucis communs d'un couple au sujet d'un enfant signifient en général un lien qui doit justement mener plus loin que cet enfant, et qui est souvent fait de sympathies qui ne peuvent absolument pas se passer d'un stade intermédiaire tel que celui-là. (Ibid., p.132)

On le voit bien, le rôle du tiers est donc de créer une unité entre deux éléments. Notons de plus que les deux exemples et les deux formes de lien (direct et indirect) que Simmel propose renvoient bien aux deux manières de concevoir le tiers que nous avions pu dégager plus haut.

Il nous reste un dernier point à discuter avant de passer à l'étude du second article. Il s'agit de se demander comment Simmel décrit le mécanisme par lequel une tierce personne ou ce que nous appellerons pour l'instant une « entité invisible » parvient à lier les individus au-delà de leurs différences ou antagonismes individuels. Il semble que la réponse à cette question consiste en ce que le tiers doit transformer des énergies subjectives dans une forme objective. Regardons l'extrait qui nous permet d'affirmer cela :

En termes psychologiques, il s'agit de réduire la forme volontaire de l'antagonisme à sa forme intellectuelle : la raison est partout le principe de la réconciliation, sur son terrain on peut réunir ce qui reste inconciliable sur le terrain du sentiment et de la décision ultime de la volonté. La tâche du médiateur consiste donc à amener cette réduction, à la représenter en quelque sorte en sa personne, ou encore : à constituer une sorte de station centrale qui, quelle que soit la forme sous laquelle la matière du conflit y entre d'un côté, ne la restituera de l'autre côté que sous une forme objective, et gardera tout ce qui en outre entretient inutilement le conflit mené sans médiation. (Ibid., p.134)

Le tiers est médiateur. Il a pour fonction de lier les individus malgré leurs antagonismes personnels, en posant dans les actions réciproques un rapport à une objectivité qui dépasse l’individualité de celui qui l'incarne.

\subsection{Le tiers invisible}

Maintenant que nous connaissons la fonction sociale du tiers, ainsi que son mécanisme de fonctionnement, il faut voir ce qui se cache derrière ces entités invisibles que constitue cette seconde sorte de tiers. Le paragraphe du chapitre 3 de Sociologie: «Domination et subordination » intitulé «Domination d'un principe objectif impersonnel» (Ibid. pp.219-234)4 propose des éléments de réponses intéressants.

\footnotetext{
4. Ce chapitre fut comme le précédent publié sous forme d'article. Nous avons ainsi pu comparer la version de 1999 avec celle de 1896 publiée dans l'American Journal of Sociology. Il est clair que la conception du monde social que nous présentons était déjà posée par Simmel en 1896. Il semble bien que Simmel renvoie de manière claire à cet article lorsque dans le chapitre 2, il nous dit que: «cette médiation d'un troisième élément [1’Eglise]
}

Émulations - Revue des jeunes chercheurs en sciences sociales 
La réflexion que Simmel aborde dans ce chapitre porte sur les motifs de l'action humaine. Il faudra voir la part que jouent ces tiers invisibles dans la détermination des formes des actions réciproques. Cela permettra de comprendre ce que sont ces tiers invisibles. Regardons l'extrait suivant :

Le motif de l'action ne relève plus d'un pouvoir concrètement humain, même supra-individuel ; mais ici, la source des nécessités morales se place au-delà de l'opposition entre l'individu et la collectivité. (...) La libre conscience de l'homme qui agit, la raison de l'individu ne sont que leur vecteur, le lieu où elles sont à l'œuvre. Leur force impérative est issue d'elles-mêmes, de leur valeur intérieure, supra-personnelle, d'une idéalité objective que nous devons reconnaître, que nous le voulions ou non, comme une vérité qui n'a pas besoin de se concrétiser dans une conscience pour être valable. Mais le contenu qui remplit ces formes (...) c'est l'exigence sociale, qui à présent n'agit plus en quelque sorte avec son impetus social, mais en se réincarnant, comme par métempsychose, en une norme qui doit être remplie pour elle-même, et non pour moi ou pour toi. (Ibid., p. 224) (c'est nous qui soulignons)

Simmel nous dit qu'il existe des motifs de l'action qui se trouvent au-delà des individus et au-delà de la collectivité. Leur force impérative est issue d'eux-mêmes. Ils ne se manifestent que dans l'action des individus. Plus spécialement, ces motifs extérieurs aux individus et à la collectivité prennent la forme de normes.

Cette description d'un principe de détermination extérieur aux individus est sous-tendue par une vision particulière du monde social. Selon Simmel, le monde social est en effet composé de trois entités. Les individus, la société et finalement, l'objectivité. Et c'est dans cette affirmation que réside la force de l'analyse de l'auteur:

La société est souvent le tiers qui résout les conflits entre les individus et l'objectivité, ou qui établit des passerelles par-dessus les fossés qui les séparent. (Ibid., p. 224).

Cette formule veut dire que la société — c'est-à-dire l'ensemble des sociabilités, c'est-à-dire encore, l'ensemble dynamique des actions réciproques qui prennent sans arrêt des formes différentes — est le lieu où se rencontrent l'individu manifestant sa subjectivité propre et l'objectivité des normes extérieures. C'est donc dans les actions réciproques que se mêlent à la fois l'objectivité du tout et la subjectivité de l'individu. C'est ce que résume encore la formule suivante :

La société s'intercale entre l'homme individuel et la nécessité universelle de la nature; en tant que réalité psychique concrète, elle est en contact avec le premier, en tant qu'universel avec la seconde. Elle est en effet l'universel sans pour autant être abstraite. (...) Mais ce face-à-face [entre l'individu et la société] est en même temps un l'un dans l'autre, sa dure indifférence à l'égard de l'individu est en même temps un intérêt, l'objectivité sociale a besoin, sinon de telle subjectivité particulière, du moins de la subjectivité de l'individu en général. Ce sont de telles déterminations qui font de la société une entité intermédiaire entre le sujet et toute universalité ou objectivité absolument impersonnelle. (Ibid., p. 225)

Nous pouvons maintenant voir plus clairement quelle est la nature de ces tiers invisibles que Simmel identifiait dans le chapitre 2. Ces tiers sont une «idéalité objective », supra-personnelle qui constitue le monde social avec d'une part les individus, et d'autre part les actions réciproques. Leur force impérative ne dépend que d'elle-même, c'est-à-dire qu'elle ne dépend pas du jugement de l'individu. L'individu n'a pas à être d'accord ou pas, il ne peut que leur obéir. Leur vérité «n'a pas besoin de se concrétiser dans une conscience pour être valable».

constituant une société devra être traitée dans un autre contexte» (Simmel, 1999 : 131). Cet autre contexte est l'étude des formes de subordination face à un principe objectif impersonnel, telle que présentée dès 1896.

Émulations - Revue des jeunes chercheurs en sciences sociales 
Nous pouvons maintenant parvenir à délimiter clairement cette seconde acception du tiers selon Simmel. Il existe selon lui des tiers qui sont des éléments idéels et objectifs. C’est-à-dire des éléments qui n'ont pas besoin pour exister de se matérialiser dans la conscience subjective des individus, mais qui ne possèdent aucune existence en dehors de leurs actions réciproques. C'est ce que veut dire Simmel quand il nous dit que la société est une entité intermédiaire entre la subjectivité et l'objectivité. Ces tiers sont des significations objectives, des éléments idéels extérieurs aux individus qui ont un rôle de médiateur. Ils prennent la forme de normes qui participent aux motifs présidant à la formation des actions réciproques en instituant des relations spécifiques entre les individus, et par là même les rassemblent dans une unité. Ce qu'il faut noter, c'est que cette objectivité sociale n'est pas la société ou le collectif. Pour Simmel, la société c'est l'ensemble des actions réciproques. La conception du tiers qu'il pose amène donc à poser un objet d'étude particulier à la sociologie : l'étude de « la relation de l'unité qu'ils [les individus] constituent avec les centres d'intérêts [les tiers objectifs] qu'ils ont en face d'eux » (Ibid., p. 132).

\section{Holisme et tiercéïté}

Cette conception du tiers, et de la vie sociale qu'elle véhicule, n'est pas construite de manière systématique. Elle traverse l'ensemble de l'œuvre de Simmel, mais Simmel, à son habitude, ne l'a pas théorisée de manière aboutie. Il est alors important, afin de rendre opératoire cette problématisation de la vie sociale, de la pousser jusqu'au bout. Nous montrerons donc, à partir des débats sur la pertinence de l'adoption d'un point de vue holiste sur la société, que toute relation sociale est — ou peut-être vue comme - une relation triadique.

Une fois cela montré, il faudra voir comment dépasser les lacunes de la conception simmelienne du tiers. Nous nous attacherons ainsi à écarter le flou régnant sur la notion de "principe objectif impersonnel », pour voir qu'elle renvoie à diverses réalités, non identifiées par notre auteur, toutes susceptibles de jouer le rôle du tiers médiateur dans une action réciproque.

\subsection{L'action sociale triadique}

Déplaçons donc le point de vue pour nous intéresser non plus directement aux tiers, mais bien aux actions sociales (que dans la tradition simmelienne nous appellerons actions réciproques). La question à laquelle il faudra répondre sera alors la suivante: Pourquoi toute action réciproque peut être vue comme une relation triadique, impliquant donc toujours trois éléments?

Nous mobiliserons pour y répondre les débats relatifs à la pertinence d'une analyse holiste de la société. Débats qui ont été synthétisés avec force par Vincent Descombes dans son livre L'institution du sens. Tout l'enjeu de ces débats repose dans la détermination de ce qui, dans la vie des hommes, est collectif. Toute la question est de savoir, d'une part, s'il existe un «Tout» social extérieur aux individus, et d'autre part, de situer ce tout. La réponse que nous allons adopter est la réponse portée par la philosophie pragmatique développée notamment par Peirce. Elle consistera à dire que ce qui est commun, ce qui dépasse l'individu, ne peut être que quelque chose de mental. Cette réponse consiste à dire que toute action réciproque contient des éléments mentaux, et que c'est dans ces éléments mentaux que se trouvent des éléments extérieurs aux individus. Dans cette perspective, toute action réciproque est triadique, le troisième élément de la relation étant toujours l'élément mental. En d'autres termes, la réponse de Peirce à la question du holisme consiste à dire que la description physique d'une action réciproque n'épuise pas tout ce qui est arrivé, car toute action réciproque comporte des éléments mentaux. En ce sens, toute action réciproque est triadique. C'est ce que Descombes nous explique quand il nous dit que :

Pour trouver une triade, nous devons sortir du positif (correspondant à ce qui est et sera) et entrer dans le conditionnel (ce qui serait, le would be). Est-ce à dire que les triades soient des zombies, des fictions sans puissance ? Peirce répond : même si le monde positif ne contient pas de triades, il peut être gouverné par des triades. Sans doute, il faudrait dire que pour lui il ne peut être

Émulations - Revue des jeunes chercheurs en sciences sociales 
gouverné que par des triades (pas par la force brute). (...). La description physique (par des faits bruts ou dyadiques) ne restitue pas tout ce qui est effectivement arrivé. (Descombes, $1996: 222$ ).

Dans une telle conception, le tiers est un élément mental. Cependant, en disant cela, nous ne répondons pas à la question du « où ». Où se trouvent les tiers ? En suivant toujours la conception pragmatique de l'action, nous dirons que ces tiers, ces éléments mentaux sont extérieurs aux individus, c'est en ce sens que notre approche des actions réciproques est holiste. Mais si ces éléments ne se trouvent pas dans les individus, ni dans leur tête, où se situent-ils? La réponse de Descombes consistera à dire que :

Ce qui sert de critère du mental, ce n'est pas l'intériorité, c'est la signification. (Descombes, $1996: 10)$

Cela revient à dire que la seule manière d'identifier et d'analyser une idée, c'est d'identifier et d'analyser l'expression qu'elle peut recevoir. En suivant la conception de Peirce, nous dirons avec Descombes que le sens d'une pensée n'est pas dans la tête de l'individu, mais dans son action. Adaptée à la société, cette thèse consiste à dire que ce qui est collectif dans une société :

C'est alors l'action de plusieurs individus, ce n'est pas le sujet de cette action. (Descombes, 1996 : 154).

C'est donc dans l'action que se donne à voir le sens d'une action. Pour comprendre cela, nous pouvons prendre l'exemple d'un homme (Pierre) qui offre un bouquet de roses à une femme (Ariane) ${ }^{5}$. Lorsque Pierre, invité par Ariane à dîner, lui offre des fleurs, il ne demande pas simplement à Ariane d'accepter ses fleurs, il lui demande aussi de reconnaitre qu'il lui offre des fleurs avec tout ce que cela signifie (c'est-à-dire qu'il désire, par exemple, lui montrer de la reconnaissance d'être invité à dîner). Il demande à Ariane de reconnaître qu'il agit comme donnant des fleurs et donc d'agir comme tel. Cette reconnaissance ne dépend pas d'un accord entre Pierre et Ariane, cet accord que demande Pierre n'est pas mental, il n'est pas comme un contrat, il ne résulte pas d'un dialogue entre Pierre et Ariane. Cet accord implique un partage de significations entre Pierre et Ariane, mais aussi ente Pierre, Ariane et tout les membres de la communauté dans laquelle on offre des fleurs.

Il est très clair que, pour que cette action réciproque d'offrir des fleurs ait lieu, il faut que les deux parties prenantes se réfèrent à un troisième élément commun, extérieur à eux, qui va permettre aux individus de donner du sens à cette action, de la comprendre. Nous dirons, toujours dans une perspective pragmatique, que ce troisième élément mental doit être perçu comme une norme et non pas comme un fait contraignant.

La différence est importante. Dire que ces tiers sont des normes, c'est affirmer que les actions réciproques sont toujours contingentes. C'est dire que la forme de deux actions réciproques ayant la même signification, ne sera jamais la même. Ainsi, il existe vraisemblablement une infinité de manières d'offrir des fleurs, car chaque action réciproque est toujours colorée par l'individu qui agit. Les actions humaines sont contingentes, parce que, comme le montre Wittgenstein, il ne peut jamais exister de règles qui disent comment suivre une autre règle. Considérer ces tiers comme des normes permet donc d'introduire l'idée chère à Joas (Joas, 1999) que l'agir est toujours un agir créationnel, l'action est sociale, mais elle n'est pas que sociale.

Le corrélat de cela est, comme le montre Gély, qu'une action réciproque est toujours susceptible d'échouer. L'individu, en voulant agir, peut ne pas saisir correctement le tiers. Ainsi, par exemple, Pierre, lors d'un voyage, se retrouve sur une île perdue dans le Pacifique où vit une jolie demoiselle de qui il s'éprend. Pour lui témoigner son affection, il décide de lui offrir un bouquet de fleurs. Or, il se fait que sur cette île, on n'offre pas de fleurs ; l'action de Pierre ne sera pas comprise et échouera, la demoiselle ne comprendra pas qu'il lui témoigne de l'intérêt. Cette action échoue, car le tiers signifiant

5. Nous reprenons cet exemple à Raphaël Gély : (Gély, 2005)

Émulations - Revue des jeunes chercheurs en sciences sociales 
commun de référence fait défaut. La demoiselle ne comprendra pas son rôle dans cette action, car l'action d'offrir des fleurs est réciproque au sens où elle comporte deux rôles qui ne peuvent aller l'un sans l'autre. Il faut un donneur et un receveur. Puisque dans notre cas, la jeune fille n'est pas receveuse, Pierre n'offre pas de fleurs.

Cette conception pragmatique du tiers et de la vie sociale constitue une radicalisation de la conception simmelienne du monde social présentée plus haut. Elle nous mène en effet à dire que toutes les relations entre individus doivent être considérées comme triadiques. Et ce serait en ce sens que les relations sociales sont toujours des actions réciproques, car, pour qu'elles aient lieu, il faut que les différents individus pris dans l'interaction définissent réciproquement la signification de leur action individuelle à partir de tiers communs. Comme le montre Simmel, le social ne se donne à voir que dans l'action. En ce sens, ce sont les actions réciproques qui sont sociétés. La radicalisation qu'il s'agit d'effectuer ici consiste à dire que cette conception est l'exemple paradigmatique d'une conception holiste de la vie sociale. Ainsi, avec Descombes, il s'agit de dire que ce que doit étudier en dernier lieu une analyse holiste de la société c'est :

[Le] rapport de délimitation et de définition réciproque entre parties. (Descombes, 1996 : 184)

Ce à quoi doit s'intéresser une analyse holiste, c'est aux relations réciproques qui lient les parties d'un tout, ce n'est pas aux parties ni au tout. Car:

C'est l'interdépendance des éléments qui est à l'origine même de la notion même d'attribut holiste. (Ibid., p. 154)

La ressemblance avec les perspectives de l'analyse simmelienne est frappante. Quand Simmel nous dit que la sociologie doit étudier les actions réciproques des individus, il ne nous dit pas autre chose que ce que Descombes nomme une analyse holiste de la société. Si une analyse holiste consiste en l'étude des rapports de délimitation et de définition réciproque entre les parties d'un tout, Simmel pose de manière paradigmatique les jalons d'une analyse holiste. En disant que la société est le médiateur entre les individus et l'objectivité sociale.

\subsection{Société et intersubjectivité}

La conception du tiers que nous avons posée à partir des travaux de Simmel possède encore une lacune qu'il nous faut résoudre. Pour l'instant, nous disons avec Simmel que les tiers sont des significations partagées, des normes, ou encore des principes objectifs qui sont nécessaires à l'élaboration de liens entre les individus. La question est de savoir comment rendre compte de ces tiers, comment leur faire une place dans une analyse de la société. En d'autres termes, il faut se demander quel statut accorder à ces significations communes ? Il semble en effet que Simmel place derrière cette appellation de principe objectif impersonnel un ensemble de réalités qui n'ont pas grand chose à voir les unes avec les autres. Il donne ainsi comme exemple de tiers les soucis communs d'un couple au sujet d'un enfant (Simmel, 1999 : 138), l'Eglise (Ibid., p.131), la vérité (Ibid. p., 226) ou encore la justice (Ibid. p., 227). Autant de réalités qui ne sont pas clairement du même ordre. Ce que nous savons, c'est que toutes ces réalités sont des significations extérieures aux individus (quoique l'Eglise possède une existence matérielle, mais est traitée par Simmel comme un tiers en ce qu'elle instaure un lien entre tous les croyants qui deviennent tous égaux en se positionnant face à un Dieu pure idéalité). Le travail qu'il nous faut effectuer est donc de distinguer divers types de significations partagées qui pourront servir de tiers dans l'interaction.

A ce titre, nous trouverons à nouveau une aide précieuse dans les travaux de Descombes, avec qui nous pouvons distinguer, au sein des significations communes, divers types de significations. En reprenant les analyses de Charles Taylor, qui propose de distinguer les significations communes en tant que telles des significations intersubjectives. Cette distinction est importante car elle va nous permettre de distinguer différentes sortes d'actions réciproques.

Émulations - Revue des jeunes chercheurs en sciences sociales 
Les significations intersubjectives correspondent :

Aux phénomènes de consensus entre des sujets indépendants. Un bon exemple est celui du goût. Une enquête d'opinion peut montrer que la tendance du public se porte vers tel modèle de consommation; tel costume, tel loisir. Cela veut dire que bien des gens (...) expriment les mêmes préférences, se prononcent dans le même sens, répondent de la même façon au questionnaire qui leur est soumis (...). Il s'agit [l'accord des jugements de goût] en effet d'une convergence entre des jugements que chacun forme librement, mais d'une convergence qui n'a rien de fortuite. Le jugement de goût a donc deux caractéristiques remarquables. D'une part quelqu'un qui veut porter un jugement de goût veut justement faire preuve de goût et non enregistrer une simple réaction idiosyncrasique de sa part. (...) il en appelle à un sensus communis, à une forme humaine de sensibilité. Pourtant, l'invocation d'un tel universel ne saurait dispenser le sujet de porter lui-même ses jugements esthétiques. (Descombes, 1996 : 292).

Une première sorte de signification partagée instaurant un lien entre des individus est donc la signification intersubjective. Il s'agit d'un jugement formé par des agents autonomes. Ce jugement n'est cependant pas une signification purement personnelle, puisqu'il rentre en convergence avec le jugement des autres. Il y a donc par ce fait création d'un lien entre les personnes porteuses de tels jugements, du fait que ceux-ci soient communicables et discutables. Ces jugements partagés impliquent donc la création d'une relation à autrui, mais, cette relation n'est qu'une possibilité de relation ; on parlera d'une relation intersubjective mais pas encore de société. La forme de cette relation n'est pas contrainte par l'imposition d'une signification extérieure commune. Elle ne place pas les individus dans des rôles complémentaires.

A côté de ce premier type de signification partagée, il en existe un second qui sera appelé les significations communes. Celles-ci sont des significations dont « la communauté ne relève pas d'un consensus intersubjectif» (Ibid., p. 293). Ces significations communes ne sont pas des points communs que l'on découvrirait en regardant dans les têtes des individus. Ce sont des significations instituées. C'est-àdire qu'elles sont publiques et sociales. Ces significations sont inculquées aux individus de façon à rendre possibles des conduites coordonnées du point de vue du groupe. On suppose en disant cela qu'il existe un sens impersonnel et général de la pratique, qui existe en dehors de l'individu. Ces significations se trouvent dans les pratiques et sont essentiellement des modes de relation sociale et d'action réciproque. Quand on offre un bouquet de fleurs à quelqu'un, le donateur, comme le receveur, savent ce qu'implique une telle action. Ils vivent dans un monde où le sens de cette action leur préexistait et peut être mobilisé par chaque individu pour agir. Il y a dans l'action de donner un bouquet de fleurs un sens partagé par les deux parties, mais qui n'est pas de l'ordre d'un consensus intersubjectif entre ces deux parties. Ce sens n'est pas communicable de la même façon qu'un jugement intersubjectif. De la même manière qu'il ne peut exister de langage privé, il ne peut exister de manière d'offrir de fleur privée. Si Pierre décide pour déclarer sa flamme d'offrir à Ariane un sac de pommes de terre, cette action, même si Pierre la pense équivalente, n'équivaudra pas au fait d'offrir un bouquet de fleurs dans notre société. Par contre, Pierre peut très bien trouver magnifique le bouquet qu'il offre alors que sa compagne, au fait des dernières tendances le trouvera sans aucun charme. Les significations communes instituent des formes de relations particulières, mais la présence de significations communes dans une action n'empêche pas la présence de significations intersubjectives.

Cette distinction entre deux types de significations partagées est intéressante car elle nous permet de distinguer deux types de relations sociales en fonction du tiers qui sera mobilisé pour la fonder. Descombes a bien noté cela dans un article ultérieur à L'institution $d u$ sens. Voilà comment il y définit ces deux types de relations :

1/ Une relation intersubjective s'établit entre deux sujets à travers des actes indépendants les uns des autres sur le modèle dialogique : dans un dialogue, je dis quelque chose pour être entendu, je pose une question pour qu'on me réponde, mais le seul fait de parler ou de questionner ne suffit évidemment pas à faire que quelqu'un m'entende ou me réponde.

Émulations - Revue des jeunes chercheurs en sciences sociales 
2/ Une relation sociale s'établit entre des personnes lorsqu'elles coopèrent dans les conditions suivantes : la première a accompli sa part d'un acte social quand la seconde en a accompli la part complémentaire. Par exemple, entre le professeur et l'élève, la relation est seulement intersubjective quand elle est seulement tentative de communiquer, mais elle est sociale quand elle est une relation d'enseignement, car le professeur n'a enseigné que si quelqu'un a été enseigné, et un élève n'a reçu un enseignement que si quelqu'un d'autre le lui a donné. (Descombes, $2001:$ 154-155)

Cette distinction implique qu'on considère qu'il y ait dans les faits sociaux des éléments plus ou moins sociaux. Ici, ce qu'il y a de spécifiquement social dans le social, c'est l'institution. Qui ajoute à une relation liant deux subjectivités une signification totalement extérieure aux individus et qui va cependant se manifester dans leur action. En ce sens, une interaction, ou une action réciproque est une unité dyadique, qui, prise dans un système d'institution, devient une triade. C'est en ce sens que l'on pourra dire que le sujet d'une institution est toujours un sujet dyadique. Ce qu'il est important de voir dans cette conception, c'est qu'un individu accomplissant un acte social manifeste à la fois un esprit objectif, extérieur à lui, indiscutable, et un esprit subjectif. Nous sommes au plus près des thèses de Simmel.

L'avancée que permet l'étude de Descombes est qu'elle nous permet de dire que chaque action réciproque qui se joue procède en fait d'une double réciprocité. Les actions sociales sont doublement réciproques.

Dans un premier sens, la réciprocité se manifeste comme l'obligation, pour qu'une personne réussisse une action, qu'une autre personne prise dans le rôle complémentaire soit le second terme de la relation dyadique. Ainsi l'action d'enseigner est une action réciproque de ce type, puisque pour qu'il y ait un enseignant qui réussisse à enseigner, il faut de facto un étudiant qui soit enseigné. L'un ne peut aller sans l'autre. On parlera de réciprocité institutionnelle. Le tiers liant les deux individus est l'institution. Il y a réciprocité parce qu'un enseignant ne peut pas enseigner s'il n'y a pas d'étudiant pour être enseigné, et vice-versa. La réciprocité provient du fait que les deux individus sont pris dans un système d'action commun.

Dans un second sens, les interactions sont marquées de réciprocité au sens où chaque action réciproque est guidée par des jugements intersubjectifs qui correspondent à tous les jugements que nous pouvons émettre et qui sont l'expression de notre subjectivité propre. Ceux-ci peuvent cependant en venir à rejoindre les jugements d'autres individus en tant qu'ils sont communicables et discutables. Les jugements intersubjectifs par excellence sont les jugements de goût, qui sont toujours l'expression de ma subjectivité mais en même temps se rapportent à l'existence d'un jugement partagé dans une communauté. Ces jugements impliquent donc des actions réciproques au sens plus spécifique où chaque personne en formulant un tel jugement influence les jugements des autres et donc leur action. Nous parlerons de réciprocité dialogique. Le tiers ici est un jugement subjectif partagé, c'est-à-dire un jugement intersubjectif. La réciprocité provient du fait que chaque partie prenant part à l'interaction est capable d'exprimer sa subjectivité et d'influencer par là la forme des actions des autres, mais en même temps, est capable d'adapter son action face aux jugements des autres.

\section{Bibliographie}

Autier J-Y. (1998), «Mobilité et processus de gentrification dans un quartier réhabilité du centre de Lyon », in : Grafmeyer Y., Dansereau F. (dir.), Trajectoires familiales et espaces de vie en milieu urbain, Lyon, Presses universitaires de Lyon, pp. 335-355.

Autier J-Y. (2003), « La gentrification du quartier Saint-Georges à Lyon, un côtoyement de mobilités différenciées ", in Bidou-Zachariasen, Retour en ville, Paris, Descarte et Cie, pp. 74-107.

Émulations - Revue des jeunes chercheurs en sciences sociales 
Bidou-Zachariasen C. (Dir.) (2003), Retour en ville, Paris, Descartes et Cie.

Benoist J.(2001), «Intersubjectivité et socialité : la phénoménologie et la question du tiers », in Benoist J. et Karsenti B. (dir.), Phénoménologie et sociologie, Paris, PUF, pp. 19-43.

Berten A. (2005), «Du tiers au Tiers » Lebrun J-P. et Volckrick E., Avons-nous encore besoin d'un tiers?, Ramonville Saint-Agne, Edition Erès.

Caplow T (1971), Deux contre un. Les coalitions dans la triade (1968), Paris, Colin.

Choay F.(1994), «Le règne de l'urbain et la mort de la ville », in : Dethier J., Guiheux A. (dir.), La ville, art et architecture en Europe, 1870-1993, Paris, Centre G. Pompidou, pp. 26-35.

Descombes V.(1996), Les institutions du sens, Paris, Les éditions de Minuit.

Descombes V (2001), «Relation intersubjective et relation sociale », in Benoist J. et Karsenti B. (dir.), Phénoménologie et sociologie, Paris, PUF, pp. 127-157.

Donzelot J.(2004), «La ville à trois vitesses : relégation, périurbanisation, gentrification » in Esprit, La ville à trois vitesses, mars-avril, $\mathrm{n}^{\circ} 3-4$, pp. 14-40.

Francq B.(2003), La ville incertaine, Politique urbaine et sujet personnel, Louvain-la-Neuve, BruylantAcademia.

Gély R. (2005), «Les Usages du tiers», in Lebrun J-P. et Volckrick E., Avons-nous encore besoin d'un tiers ?, Ramonville Saint-Agne, Edition Erès.

Hénaff M. (2004), «Vers la ville globale : monument, machine, réseau », In Esprit, La ville à trois vitesses, mars-avril, $n^{\circ} 3-4$, pp. 244-279.

Joas H.(1999), La créativité de l'agir, Paris, Cerf.

Leloup X. (2002), La ville de l'autre, Louvain-la-Neuve, Presses universitaires de Louvain.

Martuccelli D.(2002), Grammaires de l'individu, Paris, Gallimard.

Mongin O. (2005), La condition urbaine, La ville à l'heure de la mondialisation, Paris, Seuil.

Simmel G. (1896), «Superiority and subordination as subject-matter of sociology », The American Journal of Sociology, vol 2, pp 167-189; 392-415.

Simmel G. (1902), "The number of members as determining the sociological forme of the group ", The American Journal of Sociology, vol 8, pp 1-46 ; 158-196.

Simmel G. (1999), «La détermination quantitative du groupe », in. Simmel G., Sociologie. Etudes sur les formes de la socialisation [1908], Paris, PUF, pp. 81-161.

Simmel G. (1999), «Domination et subordination », in. Simmel G., Sociologie. Etudes sur les formes de la socialisation [1908], Paris, PUF, pp. 161-265.

Van Campenhoudt L. et al (2005), La méthode d'analyse en groupe, Paris, Dunod.

Émulations - Revue des jeunes chercheurs en sciences sociales 